International Journal of Pure and Applied Mathematics

Volume 103 No. 2 2015, 159-162

ISSN: 1311-8080 (printed version); ISSN: 1314-3395 (on-line version)

url: http://www.ijpam.eu

doi: http://dx.doi.org/10.12732/ijpam.v103i2.2

\title{
SOLUTION OF THE DIOPHANTINE EQUATION
}

$$
\left(2^{k}-1\right)^{x}+\left(2^{k}\right)^{y}=z^{2}
$$

Alongkot Suvarnamani $^{1}{ }^{\S}$, Tawan Ampawa ${ }^{2}$

${ }^{1,2}$ Department of Mathematics

Faculty of Science and Technology

Rajamangala University of Technology Thanyaburi

Thanyaburi, Pathum Thani, 12110, THAILAND

\begin{abstract}
In this paper, we will apply the Catalan's conjecture to find the solution of the Diophantine equation $\left(2^{k}-1\right)^{x}+\left(2^{k}\right)^{y}=z^{2}$ where $k$ is an odd integer at least 3 .
\end{abstract}

\section{AMS Subject Classification: 11D61}

Key Words: Diophantine equations, exponential equations

\section{Introduction}

In 1844, Catalan [1] posed a conjecture that $(a, b, x, y)=(3,2,2,3)$ is a unique solution of the Diophantine equation $a^{x}-b^{y}=1$ where $a, b, x$ and $y$ are integers with $\min \{a, b, x, y\}>1$. Then Mihailescu [2] proved the Catalan's conjecture in 2004.

In 2011, Suvarnamani, Singta and Chotchaisthit [6] proved that two Diophantine equations $4^{x}+7^{y}=z^{2}$ and $4^{x}+11^{y}=z^{2}$ have no non-negative integer solution.

In 2012, Suvarnamani [5] found that the Diophantine equation $A^{x}+B^{y}=$ $C^{z}$ has some non-negative integer solutions. Then Sroysang [4] proved that $(0,1,3)$ is a unique non-negative integer solution of the Diophantine equation $7^{x}+8^{y}=z^{2}$.

Received: September 17, 2014

(C) 2015 Academic Publications, Ltd.

${ }^{\S}$ Correspondence author 
In 2014, Simtrakankul [3] fonnd that the Diophantine equation $\left(2^{k}-1\right)^{x}+$ $\left(2^{k}\right)^{y}=z^{2}$ has a unique solution $\left(1,0,2^{k / 2}\right)$ where $k$ is an even integer at least 4. Then Suvarnamani [7] found the solution of the Diophantine equation $p^{x}+q^{y}=z^{2}$ where $p$ is an odd prime number which $q-p=2$ and $x, y$ and $z$ are non-negative integers. After that Suvarnamani studied in [8] about the Diophantine equation $p^{x}+(p+1)^{y}=z^{2}$ where $p$ is an odd prime number and $x, y$ and $z$ are non-negative integers.

In this paper, we will use the Catalan's conjecture to solve the Diophantine equation $\left(2^{k}-1\right)^{x}+\left(2^{k}\right)^{y}=z^{2}$ where $k$ is an odd integer at least 3 .

\section{Preliminaries}

Lemma 2.1. $(a, b, x, y)=(3,2,2,3)$ is a unique solution of the Diophantine equation $a^{x}-b^{y}=1$ where $a, b, x$ and $y$ are integers with $\min \{a, b, x, y\}>1$.

Proof. See in [4].

Lemma 2.2. Let $k$ is an odd integer at least 3 and $y, z$ are non-negative integers. Then the Diophantine equation $1+\left(2^{k}\right)^{y}=z^{2}$ has a unique solution $(y, z, k)=(1,3,3)$.

Proof. Let $k$ is an odd integer at least 3 and $y, z$ are non-negative integers such that $1+\left(2^{k}\right)^{y}=z^{2}$. We consider in 2 cases.

Case 1: $y=0$. Then $z^{2}=2$ which is impossible.

Case 2: $y \geq 1$. Then $z^{2}-2^{k y}=1$. We have $z>2$, then $\min \{z, 2, k y\}>1$. By Lemma 2.1, we get $z=3$ and $k y=3$. Hence $k=3$ and $y=1$.

Then the Diophantine equation $1+\left(2^{k}\right)^{y}=z^{2}$ has a unique solution $(y, z, k)=$ $(1,3,3)$.

Lemma 2.3. Let $k$ is an odd integer at least 3 and $x, z$ are non-negative integers. Then the Diophantine equation $\left(2^{k}-1\right)^{x}+1=z^{2}$ has no solution.

Proof. Let $k$ is an odd integer at least 3 and $x, z$ are non-negative integers such that $\left(2^{k}-1\right)^{x}+1=z^{2}$. We consider in 3 cases.

Case 1: $x=0$. Then $z^{2}=2$ which is impossible.

Case 2: $x=1$. Then $z^{2}=2^{k}$ which is impossible. 
Case 3: $x>1$. We have $\left(2^{k}-1\right)^{x} \geq\left(2^{k}-1\right)^{2}$. We have $z \geq 2$ and $2^{k}-1 \geq 4$, then $\min \left\{z, 2^{k}-1,2, x\right\}>1$. By Lemma 2.1 , we get $z=3,2^{k}-1=2$ and $x=3$. But it is impossible.

Then the Diophantine equation $\left(2^{k}-1\right)^{x}+1=z^{2}$ has no solution.

\section{Main Theorem}

Main Theorem 3.1. $(0,1,3,3)$ and $(2,2,5,2)$ are only two solutions $(x, y, z, k)$ of the Diophantine equation $\left(2^{k}-1\right)^{x}+\left(2^{k}\right)^{y}=z^{2}$ where $k$ is an odd integer at least 3 and $x, y$ and $z$ are non-negative integers.

Proof. Let $k$ is an odd integer at least 3 and $x, y$ and $z$ are non-negative integers such that $\left(2^{k}-1\right)^{x}+\left(2^{k}\right)^{y}=z^{2}$. By Lemma 2.3, we have $y \geq 1$. Then we consider in 2 cases.

Case 1: $x=0$. Then $1+\left(2^{k}\right)^{y}=z^{2}$. By Lemma 2.2, we get $(x, y, z, k)=$ $(0,1,3,3)$ is a solution of the Diophantine equation $\left(2^{k}-1\right)^{x}+\left(2^{k}\right)^{y}=z^{2}$.

Case 2: $x \geq 1$. We have $z$ as an odd integer. So, $z^{2} \equiv 1(\bmod 4)$. Then $z^{2}-2^{k y} \equiv 1(\bmod 4)$. So, we get $x$ is an even integer, i.e., $x=2 n$ where $n$ is a positive integer. Then $2^{k y}=z^{2}-\left(2^{k}-1\right)^{2 n}=\left[z-\left(2^{k}-1\right)^{n}\right]\left[z+\left(2^{k}-1\right)^{n}\right]$. So, $2\left(2^{k}-1\right)^{n}=2^{u}\left(2^{k y-2 u}-1\right)$ where $z-\left(2^{k}-1\right)^{n}=2^{u}$ and $z+\left(2^{k}-1\right)^{n}=2^{k y-u}$, for $k y>2 u$ and $u$ is a non-negative integer. Hence $u=1$, then $2^{k y-2}-\left(2^{k}-1\right)^{n}=1$.

Next, we will consider in 3 subcases.

Subcase 1: $n=1$. We have $2^{k y-2}-\left(2^{k}-1\right)=1$. Then $k=\frac{2}{y-1}$. We get $k=1$ or $k=2$. Contradiction.

Subcase $2: k y-2=1$. That is $k=3$ and $y=1$. We get $2-7^{n}=1$. It is impossible.

Subcase 3: $\min \left\{2, k y-2,2^{k}-1, n\right\}>1$. By Lemma 2.1, we get the Diophantine equation $\left(2^{k}-1\right)^{x}+\left(2^{k}\right)^{y}=z^{2}$ has no solution.

\section{Acknowledgements}

I would like to thank the referees for their comments and suggestions on the manuscript. This work was supported by the Faculty of Sciences and Technology, Rajamangala University of Technology Thanyaburi (RMUTT), Thailand. 


\section{References}

[1] E Catalan, Note extraite dune lettre adressee a lediteur, J. Reine Angew. Math., Vol. 27, (1844), 192.

[2] P. Mihailescu, Primary cycolotomic units and a proof of Catalan's conjecture, J. Reine Angew. Math., Vol. 27, (2004), 167-195.

[3] C. Simtrakankul, On the Diophantine equation $\left(2^{k}-1\right)^{x}+\left(2^{k}\right)^{y}=z^{2}$, Int. J. of Pure and Applied Math., Vol. 94, No. 1 (2014), 65-69.

[4] B. Sroysang, On the Diophantine equation $7^{x}+8^{y}=z^{2}$, Int. J. of Pure and Applied Math., Vol. 84, No. 1 (2013), 111-114.

[5] A. Suvarnamani, On the Diophantine equation of form $A^{x}+B^{y}=C^{z}$, Poceeding of RCAEM-II 2012, May 30-31, 2012.

[6] A. Suvarnamani, A. Singta and S. Chotchaisthit, On two the Diophantine equation $4^{x}+7^{y}=z^{2}$ and $4^{x}+11^{y}=z^{2}$, Sci. Technol. RMUTT J., Vol. 1, (2011), 25-28.

[7] A. Suvarnamani, Solution of the Diophantine equation $p^{x}+q^{y}=z^{2}$, Int. J. of Pure and Applied Math., Vol. 94, No. 4 (2014), 457-460.

[8] A. Suvarnamani, On the Diophantine equation $p^{x}+(p+1)^{y}=z^{2}$, Int. J. of Pure and Applied Math., Vol. 94, No. 5 (2014), 689-692. 